\title{
Study on the Deformability Characteristics of Steels for Seamless Tubes and Pipes destined for the Petrochemical Industry
}

\author{
Imre Kiss, Vasile Alexa
}

University Politehnica Timișoara, Faculty of Engineering Hunedoara, Department of Engineering \& Management, 5, Revolutiei, Hunedoara, Romania e-mails: imre.kiss@fih.upt.ro, vasile.alexa@fih.upt.ro

Abstract: The hot rolled seamless steel pipes and tubes are used successfully in areas such as the petrochemical industry (in oil and gas transport or in the extraction industry). This study includes results of the experimental hot torsion tests conducted to find the plasticity and deformability characteristics of two low alloyed medium carbon steel grades (EA < $2.5 \%)$ destined for seamless tubes and pipes manufacturing, used in the petrochemical industry (fluid transport, extraction industry): grade $43 \mathrm{MoMn} 16$ and grade $33 \mathrm{MoCr} 11$.

Keywords: low alloyed medium carbon steel grades (43MoMn16, 33MoCr11); seamless tubes and pipes; temperature; hot torsion tests; deformability; diagrams

\section{Introduction}

The major reason for the seamless steel pipe market is the rise in demand from the oil and gas industry, a sector that is expanding, and they are now being found at greater depths than before [1]. The hot rolled seamless steel pipes and tubes are used successfully in areas such as the petrochemical industry (fluid transport, extraction industry), in the construction industry (such as pipes for general use, or in installations as pipelines for gas or oil products), or in general mechanical constructions [1]. Seamless steel pipes are preferred over others due to their strength, durability and its nature to withstand extreme temperature, being used increasingly in industrial boiler applications and in the oil and gas sector [1].

The seamless pipes and tubes manufacturing process (Figure 1) involves the following two main technological processes: [2-4]

- transformation of raw materials into steel bars (electric arc furnace, ladle furnace, vacuum degassing and continuous casting processes) i.e. steel 
manufacturing (melting process, alloying, degassing, and continuous casting into bars or billets);

- transformation of steel bars into pipes or tubes, which are manufactured in different types of rolling mills, in uniformly heated in the rotary hearth heating furnace and then pierced by a piercer. In fact, the hot-rolled seamless steel pipes or tubes production base deformation process can basically be summarized as three stages:

$\equiv$ stage of perforation process (piercing in cylindrical billets into a continuous rolling mill, named piercing mill);

$\equiv$ stage of extension (elongation into a mandrel mill where the pipe or tube wall thickness is reduced by the mandrel bar and calibre rolls), and

$\equiv$ stage of finishing (diameter reducing into a sizing/reducing mill).
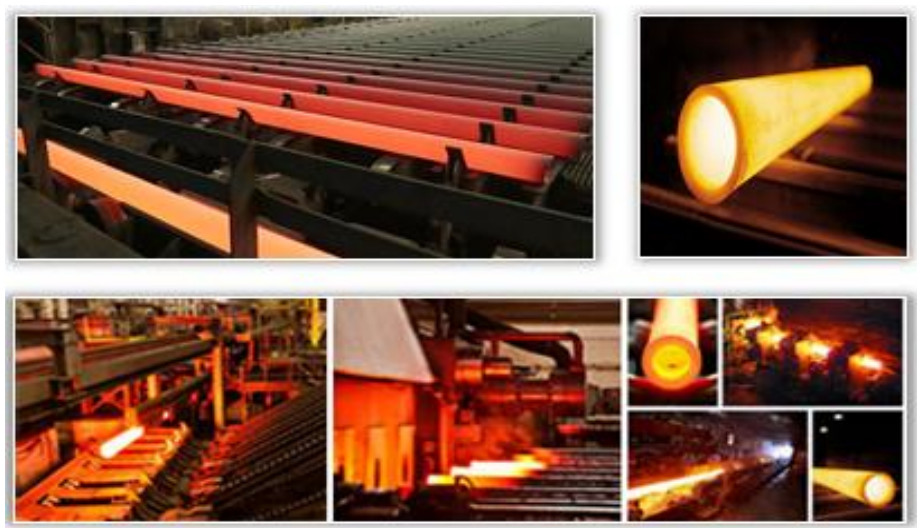

Figure 1

The seamless pipes and tubes manufacturing process

Pipes and tubes shall have smooth exterior and interior surfaces, in accordance with the manufacturing process [2-4]. The outer and inner surfaces of the pipes shall be free from cracks, overlaps of material, non-metallic inclusions or other visible defects [2-4]. All these aspects are resolved at the stage of steel manufacturing stages, the steel's chemical composition being the main technological factor that influences the quality of the half-products (steel bars and billets). There will be no discussions to such aspects in this article. Therefore, in the laboratory determinations, we will use steels that are long documented by industrial practice.

The typical rolling process (the second step of the seamless pipes and tubes manufacturing process) is schematically shown in Figure 2 [2-5]. Depending on the number of rolls, the piercing mills (stage of perforation process) can be divided into: 
- two-roll piercing mills with two working rolls and two guiding devices which hold the tube shell along the rolling axis (Figure 3a); [5]

- three-roll piercing mills with three working rolls that operate without any additional guiding devices for the metal (Figure 3b). [5]

During the second step, the steel bars or billets are subjected to large local deformations, so their deformation behaviour is very important [2-5]. In the piercing mill, deformation of the material, especially at the front end of the plug is large, and as the rolling operation progresses, the material at the centre of the plug front end is subject to an extremely large shear deformation along the plug surface [2-5].

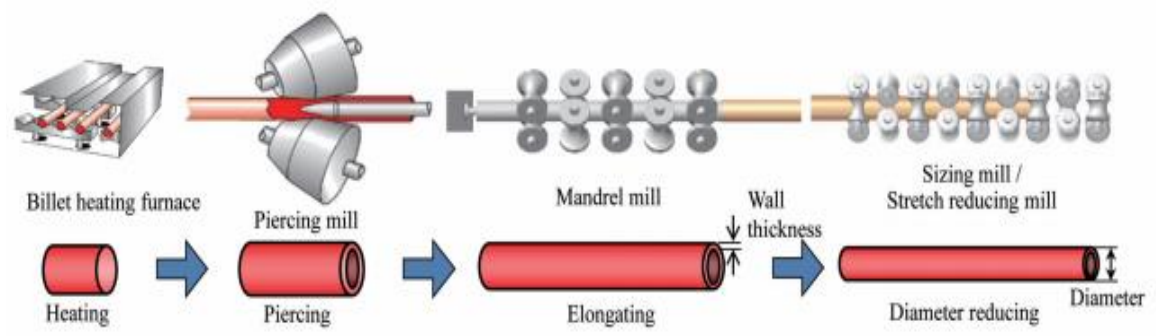

Figure 2

The typical rolling process of the seamless pipes and tubes manufacturing process - General view

(a)

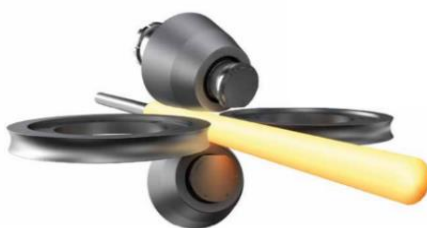

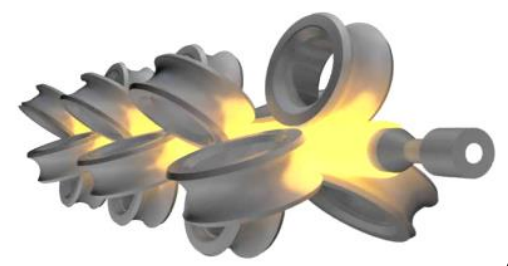

(b)

Figure 3

Typical piercing mills: (a) two-roll mills with two working rolls and two guiding devices; (b) three-roll mills with three working rolls, without any additional guiding devices

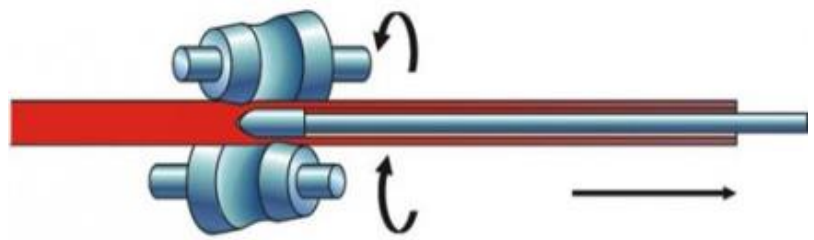

Figure 4

The two-roll piercing mills with two working rolls - General view 
The first step (stage of perforation process) is usually a matter of poking a hole through a red-hot solid billet (Figure 4) [2-5]. In fact, during the primary piercing operation, the red-hot steel billet is rolled on the cross-roll piercing mill [2-5]. The quality of the piercing process has a major effect on the downstream processes and the finished product. At this stage of the process, the deformation plays a very important role.

In the two-roll-type mandrel mill (in stage of extension), which uses two rolls on each stand, the directions perpendicular to each other within the cross-section of a tube are alternately rolled by the odd-numbered and even-numbered stands (Figure 5) [2-5]. In the stage of finishing, the pipe diameter is reduced into a sizing/reducing mill (Figure 6) [2-5].

(a)

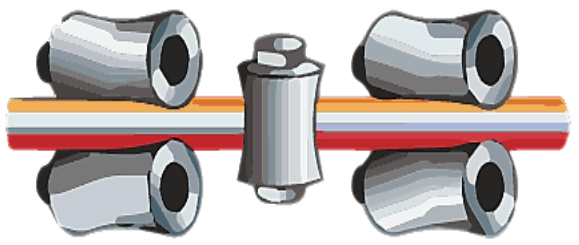

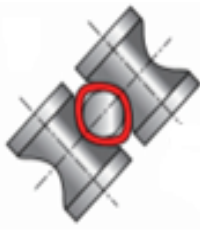

(b)

General view and cross-section of the mandrel mill (odd and even numbered stand)

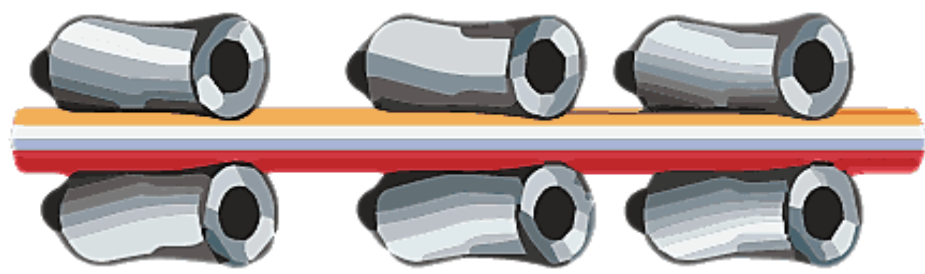

Figure 6

General view of the sizing/reducing mill and the arrangement of rolls

Knowledge of the seamless pipe and tube steel's deformability characteristics (plasticity and deformation resistance) has for the hot rollers a great practical significance because they are important elements in establishing a proper technological process [6-9].

The ability to permanently deform without breaking the internal structural bonds is defined as deformability [6-9]. In fact, the deformability is the ability of a material to be plastically deformed without the occurrence of undesired conditions (cracking of material during the plastic deformation, inadequate quality of the surface, etc.) [6-9]. In this sense, the deformability of a material is expressed by the degree of deformation to which the first cracks appear [6-9]. 
The plastic deformation of steels is based on the property of plasticity, which defines their ability to acquire permanent deformations under the action of external forces [6-9]. Therefore, knowledge of the rolled steel's plasticity becomes the determining factor in the entire hot plastic deformation.

\section{The Research Methodology}

Besides the determination of deformability characteristics (plasticity and deformation resistance), several methods enable that to study the influence of the main deformation conditions (heating rate, holding time, heating temperature, deformation rate etc.) [7-9]. For determining the deformability of the steels are used metods like: $[7,8]$

— based on the compression, rolling or forging, taking account of friction;

— based on the bending, tensile or torsion, without taking account of friction.

Among them, determination of the steel deformability by hot torsion method is the only one that allows obtaining large deformations along the length of the specimen $[7,8]$. By this method, the hot deformability of the steel is determined by subjecting to torsion a cylindrical specimen maintained at the deformation temperature in a tubular oven $[7,8]$.

The deformability caused by hot torsion reflects quite accurately the steel behavior at hot plastic deformation, and due to the fact that the specimens can be maintained in the attached oven during deformation, we can ensure the stability of temperature [7, 8]. The size of the required moment for torsion the specimen expresses the resistance to plastic deformation, and the number of torsions up to breaking expresses the plasticity limit of that steel [7, 8].

The experimental equipment used to study the steel deformability by hot torsion belongs to the Faculty of Engineering Hunedoara [7, 8]. The equipment is provided with an electric oven which provides the sample heating in the range 20$1300^{\circ} \mathrm{C}$. The experimental equipment is shown in Figure $7[7,8]$.

(a)

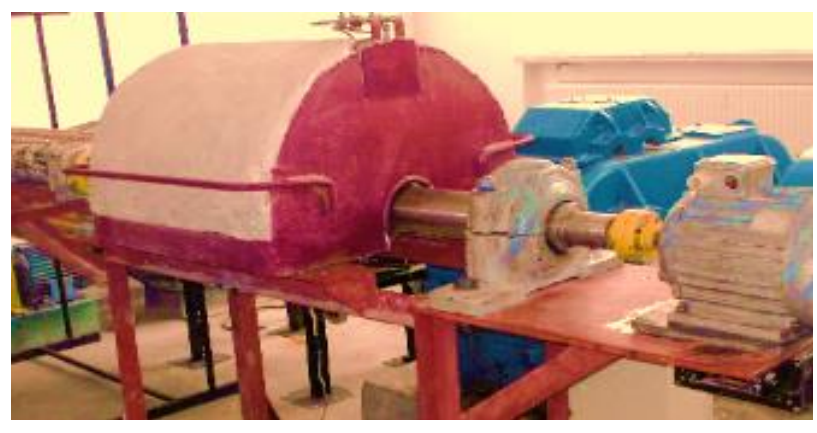


(b)

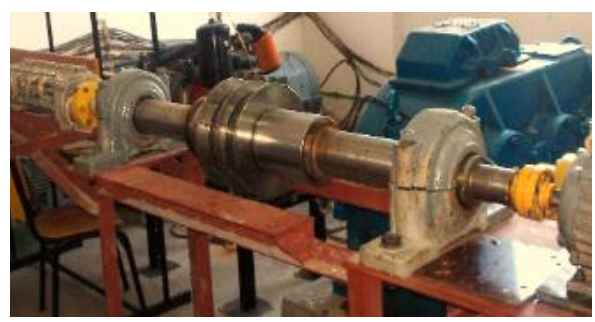

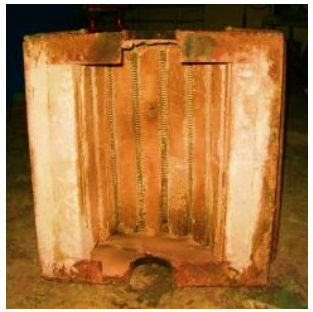

(c)

Figure 7

The experimental equipment builded for determining the hot torsion tests of steels [7, 8]

a) the prepared experimental equipment; b) the central shaft (wich fix the experimental samples), without the heating oven; c) the inside of the heating oven

The test specimens are typically cylindrical, with a calibrated small-diameter central portion, having the ration $1 / d=5$ in the point of deformation $[7,8]$. The specimens for hot torsions were mechanically taken from $\Phi 20 \mathrm{~mm}$ hot-rolled steel bars.

For the hot torsion test, we prepared 27 specimens-samples from each steel grade. They were subjected to torsional deformation by maintaining the deformation temperature in the experimental facility, from 50 to $50^{\circ} \mathrm{C}$, within the range 800$1200^{\circ} \mathrm{C}[7,8]$.

For the experimental tests, we used representative low alloyed medium carbon steel grades (43MoMn16 and 33MoCr11), destined for seamless tubes and pipes manufacturing. Typical chemical compositions of these steels are presented in Table $1[10,11]$. We used several lots of low alloyed medium carbon steel grades (two lots for $43 \mathrm{MoMn} 16$ grade - lots 1 and 2, another two lots for $33 \mathrm{MoCr} 11$ lots 3 and 4) (Table 2).
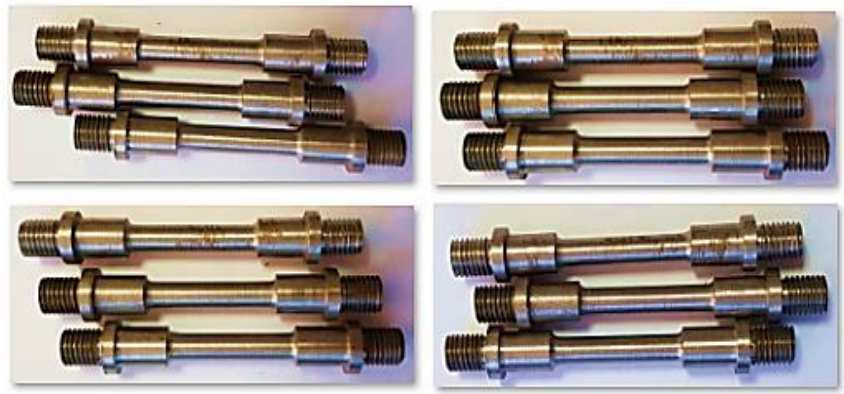

Figure 8

The steel specimens used in the hot torsion tests (experimental lots 1-4) 
Table 1

Typical chemical compositions of low alloyed medium carbon steel grades (43MoMn16 and

$33 \mathrm{MoCr} 11)$, destined for seamless tubes and pipes manufacturing [10,11]

\begin{tabular}{|l|l|l|l|l|l|l|l|}
\hline \multirow{2}{*}{ Steel grades } & \multicolumn{6}{|l|}{ The chemical composition, [\%] } \\
\cline { 2 - 8 } & $\mathrm{C}$ & $\mathrm{Mn}$ & $\mathrm{Si}$ & $\mathrm{P}$ & $\mathrm{S}$ & $\mathrm{Cr}$ & Mo \\
\hline 43MoMn16 & $0.4-0.46$ & $1.4-1.7$ & $0.17-0.37$ & 0.035 & 0.035 & - & min. 0.16 \\
\hline 33MoCr11 & $0.3-0.37$ & $0.4-0.8$ & $0.17-0.37$ & 0.035 & 0.035 & $0.9-1.3$ & $0.15-0.3$ \\
\hline
\end{tabular}

Table 2

The chemical compositions of the low alloyed medium carbon steel grades

\begin{tabular}{|l|l|l|l|l|l|l|l|l|}
\hline \multirow{2}{*}{$\begin{array}{l}\text { No. } \\
\text { lots }\end{array}$} & \multicolumn{6}{|l|}{ The chemical composition, [\%] } \\
\cline { 2 - 9 } & $\mathrm{C}$ & $\mathrm{Mn}$ & $\mathrm{Si}$ & $\mathrm{P}$ & $\mathrm{S}$ & $\mathrm{Cr}$ & $\mathrm{Mo}$ & $\mathrm{Al}$ \\
\hline 1 & 0.50 & 1.90 & 0.33 & 0.01 & 0.009 & - & 0.26 & 0.056 \\
\hline 2 & 0.42 & 1.53 & 0.38 & 0.04 & 0.008 & - & 0.26 & 0.024 \\
\hline 3 & 0.36 & 0.84 & 0.31 & 0.007 & 0.041 & 0.93 & 0.13 & 0.050 \\
\hline 4 & 0.40 & 1.00 & 0.32 & 0.007 & 0.018 & 1.16 & 0.23 & 0.069 \\
\hline
\end{tabular}

\section{The Experimental Results}

Hot torsion testing is key in find the interdependence between hot plastic deformation process parameters and the steel's properties. This method has been useful to provide the existence of hot deformability as being dependent on elevated temperatures (between $800-1200^{\circ} \mathrm{C}$ ). A versatile hot torsion testing machine (Figure 7) has been developed in the Faculty of Engineering Hunedoara, to simulate deformation schedules encountered in practical hot working operations such as rolling $[7,8]$.

The magnitude of the torque required to the specimen's torsion expresses the resistance to deformation $[7,8]$. The number of torsions up to breaking expresses the plasticity limit of that steel at a given temperature and deformation rate [7,8].

Table 3

The torque moment results of hot torsion tests: experimental lot 1 and experimental lot 2

\begin{tabular}{|c|c|c|c|c|c|}
\hline \multirow{3}{*}{$\begin{array}{l}\text { No. of } \\
\text { exp. }\end{array}$} & \multirow{3}{*}{$\begin{array}{l}\text { Testing } \\
\text { temperature, } \\
{\left[{ }^{0} \mathrm{C}\right]}\end{array}$} & \multicolumn{4}{|c|}{ Torque moment [daN $\left.{ }^{*} \mathrm{~cm}\right]$} \\
\hline & & \multicolumn{2}{|l|}{ Lot 1} & \multicolumn{2}{|l|}{ Lot 2} \\
\hline & & Unitary value & Average value & Unitary value & Average value \\
\hline 1 & \multirow{3}{*}{800} & 212 & \multirow{3}{*}{207} & 242 & \multirow{3}{*}{243} \\
\hline 2 & & 209 & & 242 & \\
\hline 3 & & 202 & & 244 & \\
\hline 4 & \multirow{3}{*}{850} & 210 & \multirow{3}{*}{191} & 168 & \multirow{3}{*}{165} \\
\hline 5 & & 200 & & 165 & \\
\hline 6 & & 163 & & 163 & \\
\hline
\end{tabular}




\begin{tabular}{|c|c|c|c|c|c|}
\hline 7 & \multirow{3}{*}{900} & 179 & \multirow{3}{*}{171} & 163 & \multirow{3}{*}{154} \\
\hline 8 & & 170 & & 149 & \\
\hline 9 & & 163 & & 131 & \\
\hline 10 & \multirow{3}{*}{950} & 134 & \multirow{3}{*}{133} & 134 & \multirow{3}{*}{136} \\
\hline 11 & & 133 & & 138 & \\
\hline 12 & & 133 & & 135 & \\
\hline 13 & \multirow{3}{*}{1000} & 119 & \multirow{3}{*}{119} & 134 & \multirow{3}{*}{134} \\
\hline 14 & & 119 & & 134 & \\
\hline 15 & & 119 & & 134 & \\
\hline 16 & \multirow{3}{*}{1050} & 104 & \multirow{3}{*}{102} & 106 & \multirow{3}{*}{107} \\
\hline 17 & & 96 & & 108 & \\
\hline 18 & & 106 & & 106 & \\
\hline 19 & \multirow{3}{*}{1100} & 85 & \multirow{3}{*}{84} & 75 & \multirow{3}{*}{76} \\
\hline 20 & & 88 & & 76 & \\
\hline 21 & & 78 & & 76 & \\
\hline 22 & \multirow{3}{*}{1150} & 79 & \multirow{3}{*}{74} & 72 & \multirow{3}{*}{72} \\
\hline 23 & & 71 & & 72 & \\
\hline 24 & & 72 & & 73 & \\
\hline 25 & \multirow{3}{*}{1200} & 40 & \multirow{3}{*}{45} & 54 & \multirow{3}{*}{56} \\
\hline 26 & & 50 & & 64 & \\
\hline 27 & & 45 & & 50 & \\
\hline
\end{tabular}

Table 4

The torque moment results of hot torsion tests: experimental lot 3 and experimental lot 4

\begin{tabular}{|c|c|c|c|c|c|}
\hline \multirow{3}{*}{$\begin{array}{l}\text { No. of } \\
\text { exp. }\end{array}$} & \multirow{3}{*}{$\begin{array}{l}\text { Testing } \\
\text { temperature, } \\
{\left[{ }^{0} \mathrm{C}\right]}\end{array}$} & \multicolumn{4}{|c|}{ Torque moment [daN*cm] } \\
\hline & & \multicolumn{2}{|l|}{ Lot 3} & \multicolumn{2}{|l|}{ Lot 4} \\
\hline & & Unitary value & Average value & Unitary value & Average value \\
\hline 1 & \multirow{3}{*}{800} & 258 & \multirow{3}{*}{257} & 242 & \multirow{3}{*}{249} \\
\hline 2 & & 251 & & 253 & \\
\hline 3 & & 262 & & 251 & \\
\hline 4 & \multirow{3}{*}{850} & 165 & \multirow{3}{*}{166} & 163 & \multirow{3}{*}{163} \\
\hline 5 & & 168 & & 163 & \\
\hline 6 & & 165 & & 163 & \\
\hline 7 & \multirow{3}{*}{900} & 150 & \multirow{3}{*}{146} & 150 & \multirow{3}{*}{151} \\
\hline 8 & & 148 & & 149 & \\
\hline 9 & & 140 & & 153 & \\
\hline 10 & \multirow{3}{*}{950} & 130 & \multirow{3}{*}{125} & 125 & \multirow{3}{*}{127} \\
\hline 11 & & 120 & & 130 & \\
\hline 12 & & 125 & & 126 & \\
\hline 13 & \multirow{3}{*}{1000} & 100 & \multirow{3}{*}{102} & 110 & \multirow{3}{*}{115} \\
\hline 14 & & 108 & & 118 & \\
\hline 15 & & 100 & & 116 & \\
\hline 16 & \multirow{3}{*}{1050} & 96 & \multirow{3}{*}{85} & 80 & \multirow{3}{*}{81} \\
\hline 17 & & 79 & & 84 & \\
\hline 18 & & 80 & & 79 & \\
\hline 19 & \multirow{3}{*}{1100} & 70 & \multirow{3}{*}{74} & 88 & \multirow{3}{*}{79} \\
\hline 20 & & 77 & & 76 & \\
\hline 21 & & 75 & & 72 & \\
\hline 22 & \multirow{3}{*}{1150} & 60 & \multirow{3}{*}{67} & 65 & \multirow{3}{*}{65} \\
\hline 23 & & 75 & & 65 & \\
\hline 24 & & 65 & & 66 & \\
\hline 25 & \multirow{3}{*}{1200} & 50 & \multirow{3}{*}{54} & 50 & \\
\hline 26 & & 57 & & 52 & 53 \\
\hline 27 & & 54 & & 58 & \\
\hline
\end{tabular}


Table 5

The number of torsions up to breaking results of hot torsion tests: lots 1 and 2

\begin{tabular}{|c|c|c|c|c|c|}
\hline \multirow{3}{*}{$\begin{array}{l}\text { No. of } \\
\text { exp. }\end{array}$} & \multirow{3}{*}{$\begin{array}{l}\text { Testing } \\
\text { temperature, } \\
{\left[{ }^{0} \mathrm{C}\right]}\end{array}$} & \multicolumn{4}{|c|}{ Number of torsions up to breaking $[-]$} \\
\hline & & \multicolumn{2}{|l|}{ Lot 1} & \multicolumn{2}{|l|}{ Lot 2} \\
\hline & & Unitary value & Average value & Unitary value & Average value \\
\hline 1 & \multirow{3}{*}{800} & 36 & \multirow{3}{*}{37} & 33 & \multirow{3}{*}{33} \\
\hline 2 & & 39 & & 33 & \\
\hline 3 & & 37 & & 33 & \\
\hline 4 & \multirow{3}{*}{850} & 39 & \multirow{3}{*}{44} & 35 & \multirow{3}{*}{38} \\
\hline 5 & & 45 & & 38 & \\
\hline 6 & & 49 & & 42 & \\
\hline 7 & \multirow{3}{*}{900} & 41 & \multirow{3}{*}{45} & 45 & \multirow{3}{*}{47} \\
\hline 8 & & 50 & & 47 & \\
\hline 9 & & 41 & & 48 & \\
\hline 10 & \multirow{3}{*}{950} & 48 & \multirow{3}{*}{46} & 46 & \multirow{3}{*}{47} \\
\hline 11 & & 44 & & 47 & \\
\hline 12 & & 45 & & 47 & \\
\hline 13 & \multirow{3}{*}{1000} & 58 & \multirow{3}{*}{52} & 52 & \multirow{3}{*}{55} \\
\hline 14 & & 46 & & 57 & \\
\hline 15 & & 52 & & 57 & \\
\hline 16 & \multirow{3}{*}{1050} & 57 & \multirow{3}{*}{59} & 68 & \multirow{3}{*}{67} \\
\hline 17 & & 58 & & 66 & \\
\hline 18 & & 65 & & 67 & \\
\hline 19 & \multirow{3}{*}{1100} & 64 & \multirow{3}{*}{63} & 70 & \multirow{3}{*}{72} \\
\hline 20 & & 66 & & 73 & \\
\hline 21 & & 59 & & 72 & \\
\hline 22 & \multirow{3}{*}{1150} & 63 & \multirow{3}{*}{64} & 71 & \multirow{3}{*}{73} \\
\hline 23 & & 64 & & 74 & \\
\hline 24 & & 65 & & 73 & \\
\hline 25 & \multirow{3}{*}{1200} & 62 & \multirow{3}{*}{68} & 71 & \\
\hline 26 & & 73 & & 78 & 74 \\
\hline 27 & & 68 & & 74 & \\
\hline
\end{tabular}

Table 6

The number of torsions up to breaking results of hot torsion tests: lots 3 and 4

\begin{tabular}{|c|c|c|c|c|c|}
\hline \multirow{3}{*}{$\begin{array}{l}\text { No. of } \\
\text { exp. }\end{array}$} & \multirow{3}{*}{$\begin{array}{l}\text { Testing } \\
\text { temperature, } \\
{\left[{ }^{0} \mathrm{C}\right]}\end{array}$} & \multicolumn{4}{|c|}{ Number of torsions up to breaking $[-]$} \\
\hline & & \multicolumn{2}{|c|}{ Lot 3} & \multicolumn{2}{|l|}{ Lot 4} \\
\hline & & Unitary value & Average value & Unitary value & Average value \\
\hline 1 & \multirow{3}{*}{800} & 41 & \multirow{3}{*}{41} & 36 & \multirow{3}{*}{ 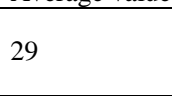 } \\
\hline 2 & & 42 & & 27 & \\
\hline 3 & & 39 & & 25 & \\
\hline 4 & \multirow{3}{*}{850} & 45 & \multirow{3}{*}{43} & 40 & \multirow{3}{*}{37} \\
\hline 5 & & 42 & & 32 & \\
\hline 6 & & 42 & & 38 & \\
\hline 7 & \multirow{3}{*}{900} & 47 & \multirow{3}{*}{47} & 47 & \multirow{3}{*}{45} \\
\hline 8 & & 49 & & 48 & \\
\hline 9 & & 44 & & 42 & \\
\hline 10 & \multirow{3}{*}{950} & 48 & \multirow{3}{*}{45} & 39 & \multirow{3}{*}{46} \\
\hline 11 & & 45 & & 50 & \\
\hline 12 & & 40 & & 48 & \\
\hline 13 & \multirow{3}{*}{1000} & 46 & \multirow{3}{*}{48} & 48 & \multirow{3}{*}{51} \\
\hline 14 & & 48 & & 55 & \\
\hline 15 & & 50 & & 50 & \\
\hline 16 & \multirow{3}{*}{1050} & 50 & \multirow{3}{*}{58} & 54 & \multirow{3}{*}{52} \\
\hline 17 & & 65 & & 50 & \\
\hline 18 & & 57 & & 50 & \\
\hline 19 & \multirow{3}{*}{1100} & 68 & \multirow{3}{*}{63} & 58 & \multirow{3}{*}{58} \\
\hline 20 & & 56 & & 54 & \\
\hline 21 & & 65 & & 60 & \\
\hline 22 & \multirow{3}{*}{1150} & 53 & \multirow{3}{*}{65} & 53 & \multirow{3}{*}{61} \\
\hline 23 & & 65 & & 60 & \\
\hline 24 & & 67 & & 69 & \\
\hline 25 & \multirow{3}{*}{1200} & 63 & \multirow{3}{*}{67} & 64 & \\
\hline 26 & & 65 & & 66 & 66 \\
\hline 27 & & 72 & & 68 & \\
\hline
\end{tabular}




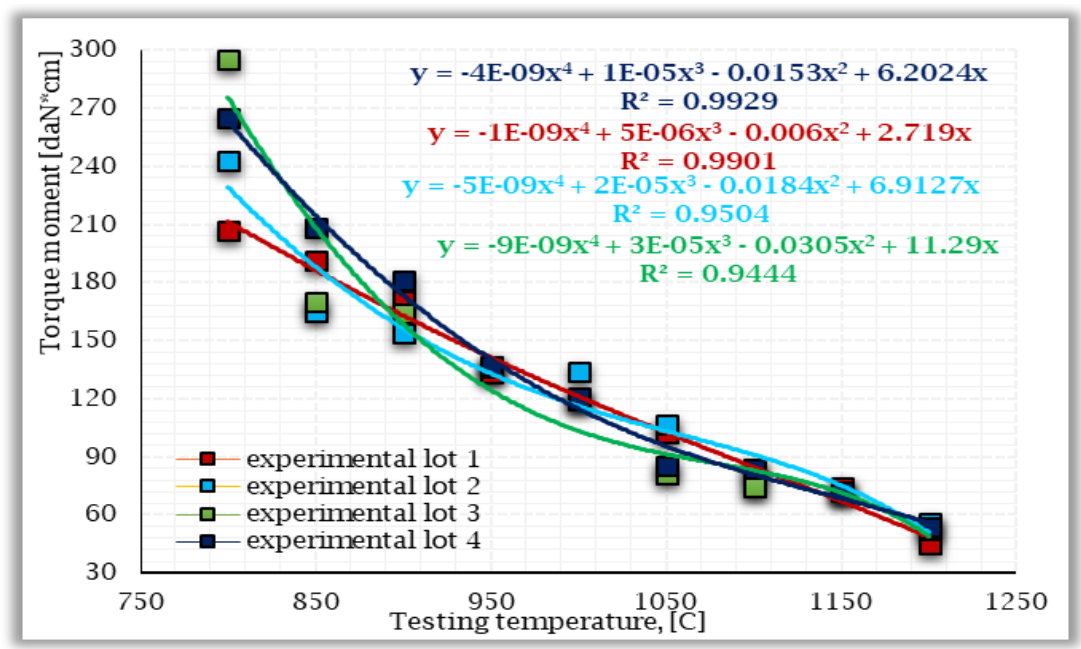

Figure 9

The variation of torque moment (variation of deformation resistance) in case of the 4 experimental lot of steels, at the experimental testing temperature (heating between $800-1200^{\circ} \mathrm{C}$ )

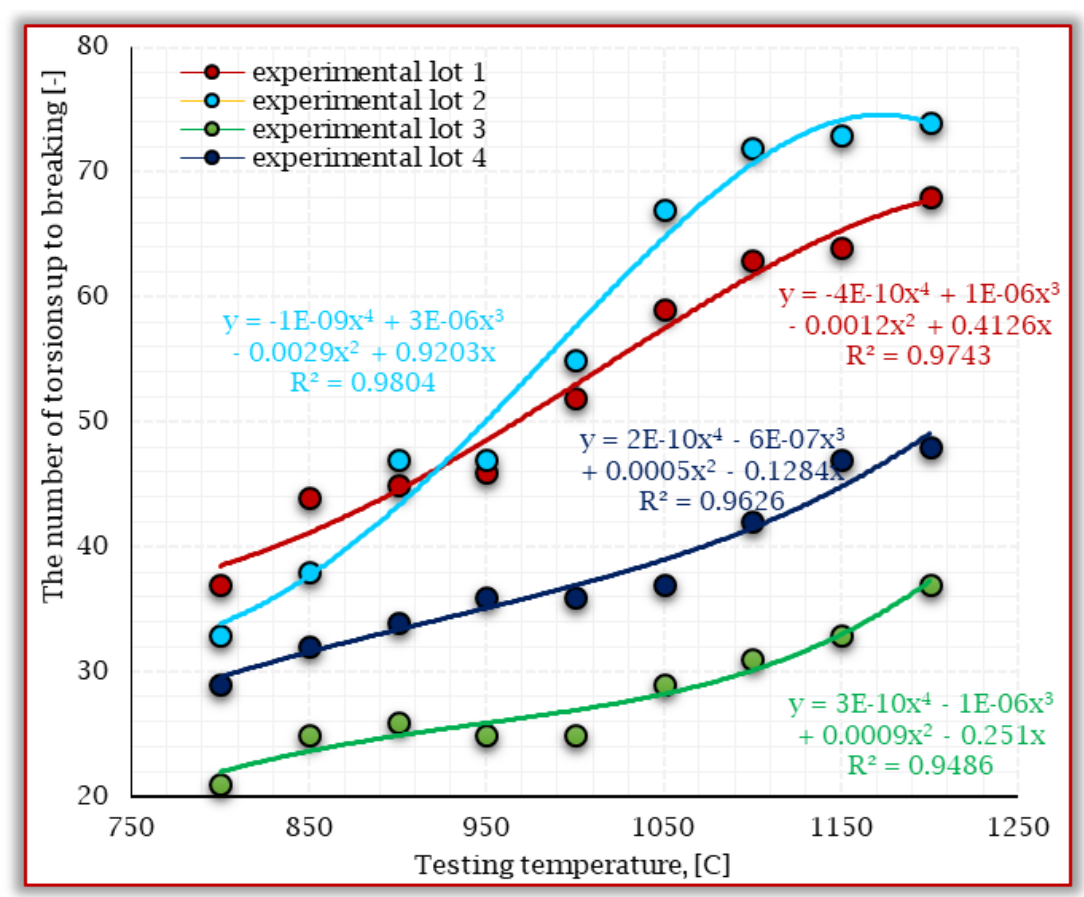

Figure 10

The number of torsions up to breaking (variations of plasticity) in case of the 4 experimental lot of steels, at the experimental testing temperature (heating between $800-1200^{\circ} \mathrm{C}$ ) 


\section{The Statistical Modeling}

Regarding the statistical modeling by the multiple linear regression, in order to understand the relationships between variables and their relevance to the problem being studied, have been established the following technical conditions:

- The used univariate and multivariate analyses were based on the statistical principle of multivariate statistics, which involves observation of the deformability characteristic's variations, in the experimental testing temperature range (heating between $800-1200^{\circ} \mathrm{C}$ ).

- In the used statistical modeling, the regression analysis is a set of statistical processes for estimating the relationships among the deformability characteristics (plasticity, described by the number of torsions up to breaking and deformation resistance, described by the torque moment value) and the testing temperature.

(a)
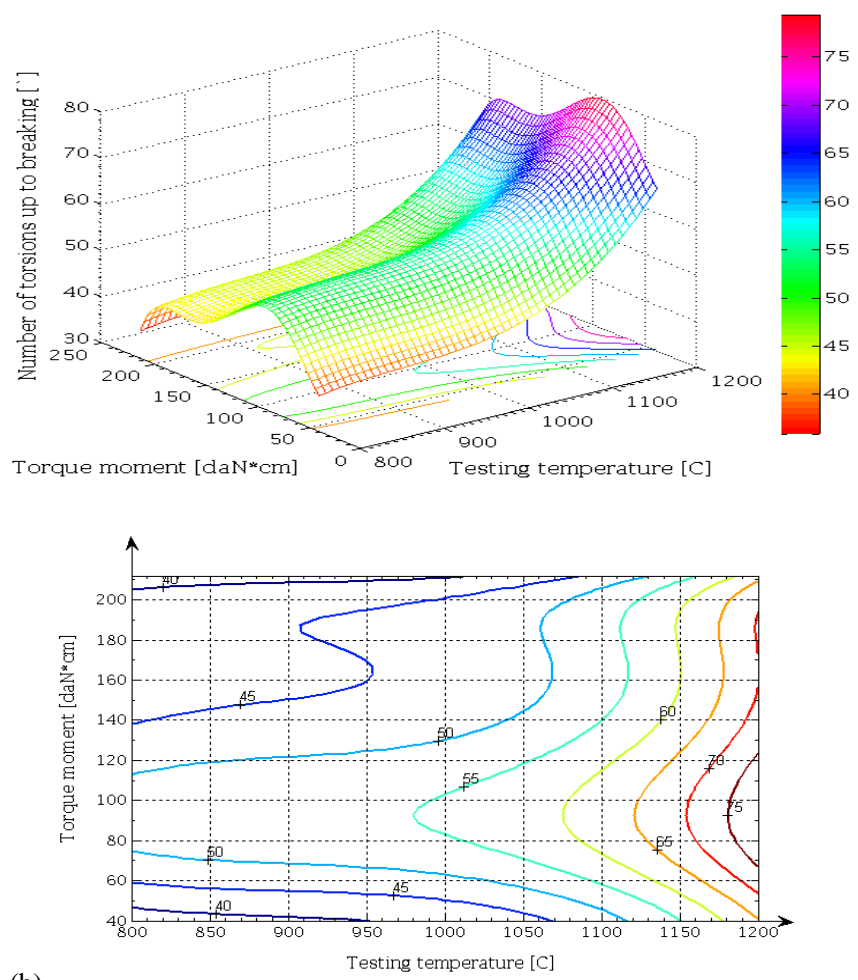

(b) 
(c)

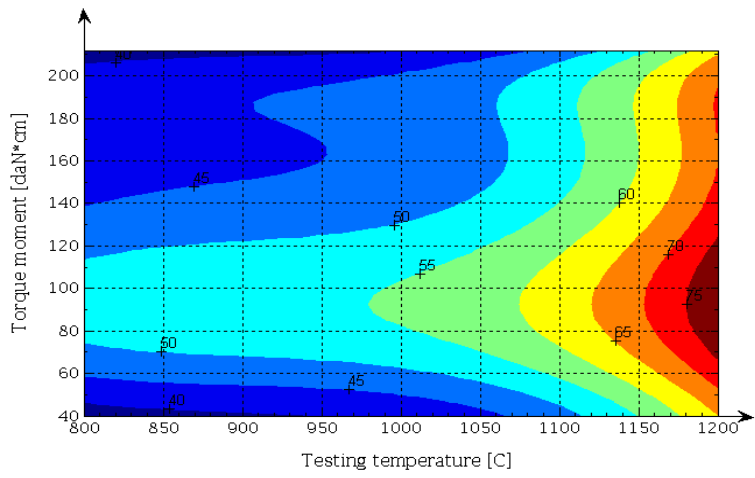

Figure 11

Diagrams for the steel specimens (lot 1), at the testing temperature (heating between $800-1200^{\circ} \mathrm{C}$ )

(a) the variation of deformation resistance, described by the torque moment value [equation type: $\mathrm{z}=\mathrm{a}_{1}$ $+a_{2} x+a_{3} x^{2}+a_{4} x^{3}+a_{5} y+a_{6} y^{2}+a_{7} y^{3}+a_{8} y^{4}+a_{9} y^{5}$, standard deviation: $r^{2}=0.8870, a_{1}=-545.8069 ; a_{2}$ $=2.0159 ; \mathrm{a}_{3}=-0.0022 ; \mathrm{a}_{4}=8.2793 ; \mathrm{a}_{5}=-2.2735 ; \mathrm{a}_{6}=0.0652 ; \mathrm{a}_{7}=-0.0007 ; \mathrm{a}_{8}=3.3254 ; \mathrm{a}_{9}=-$

$$
\text { 5.6431]; }
$$

(b), (c) the technological domains area of the deformation resistance, described by the experimental torque moment value

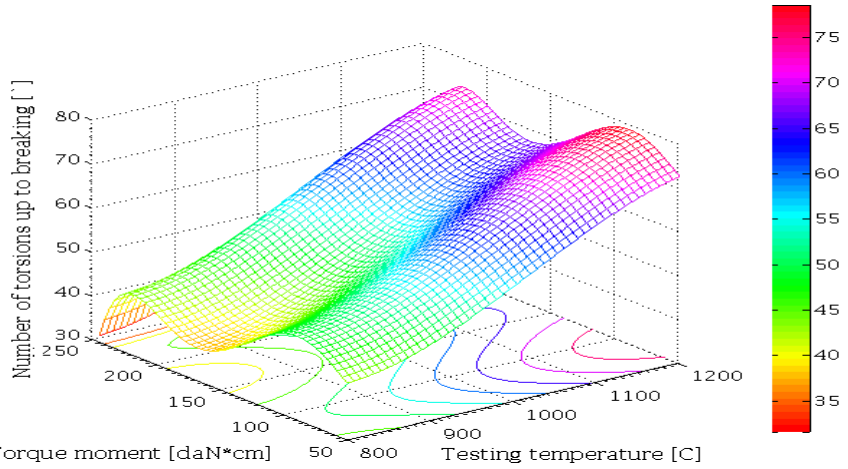

(a) Torque moment [daN* $\left.{ }^{*} \mathrm{~cm}\right] \quad 50 \quad 800 \quad$ Testing temperature [C]

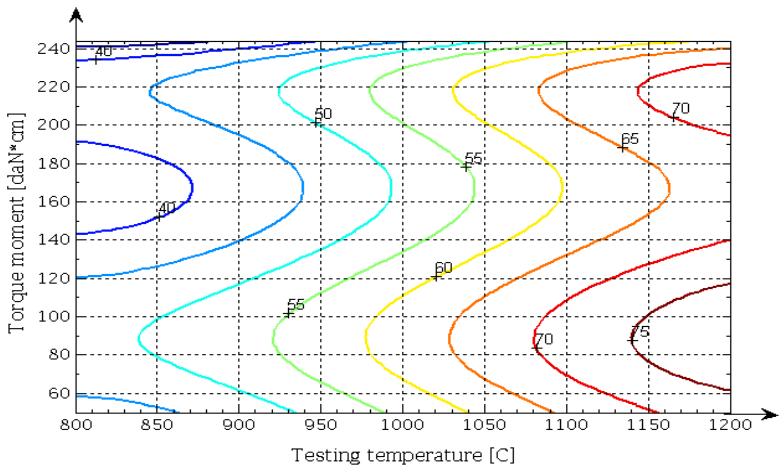




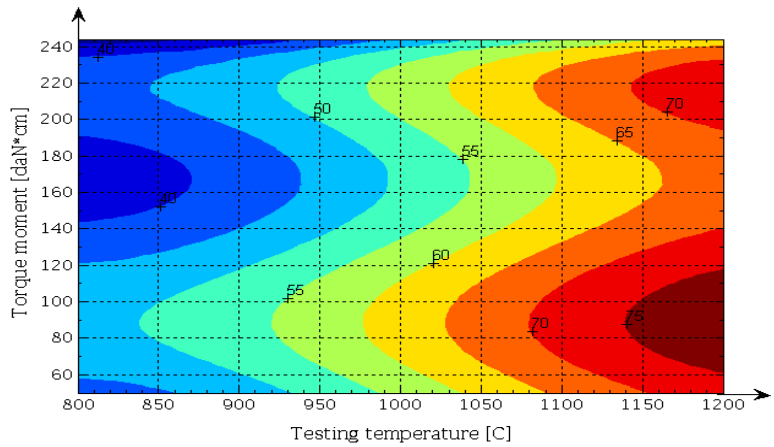

(c)

Figure 12

Diagrams for the steel specimens (lot 2), at the testing temperature (heating between $800-1200^{\circ} \mathrm{C}$ )

(a) the variation of deformation resistance, described by the torque moment value [equation type: $\mathrm{z}=\mathrm{a}_{1}$ $+a_{2} x+a_{3} x^{2}+a_{4} x^{3}+a_{5} y+a_{6} y^{2}+a_{7} y^{3}+a_{8} y^{4}+a_{9} y^{5}$, standard deviation: $r^{2}=0.9764, a_{1}=631.8348 ; a_{2}=$ $-1.8157 ; \mathrm{a}_{3}=0.0019 ; \mathrm{a}_{4}=-1.9134 ; \mathrm{a}_{5}=0.0495 ; \mathrm{a}_{6}=0.0652 ; \mathrm{a}_{7}=-0.0005 ; \mathrm{a}_{8}=2.2429 ; \mathrm{a}_{9}=-$ 3.5361];

(b), (c) the technological domains area of the deformation resistance, described by the experimental torque moment value

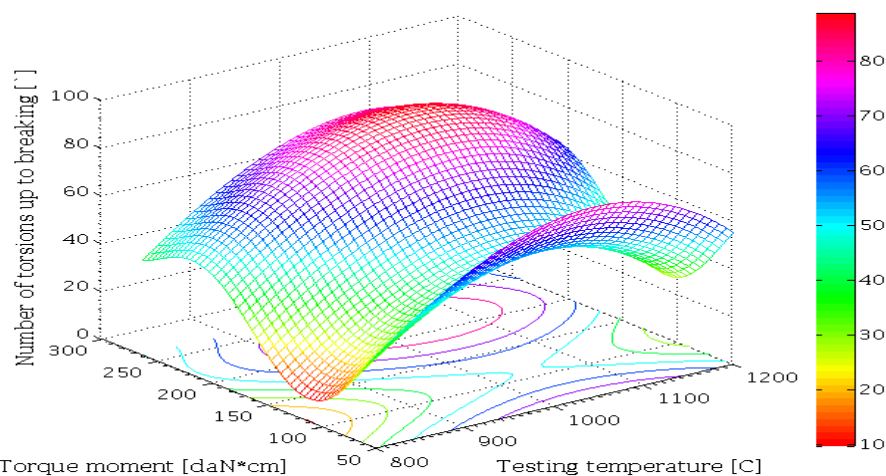

(a)

Torque moment [daN*cm] 50 soo Testing temperature [C]

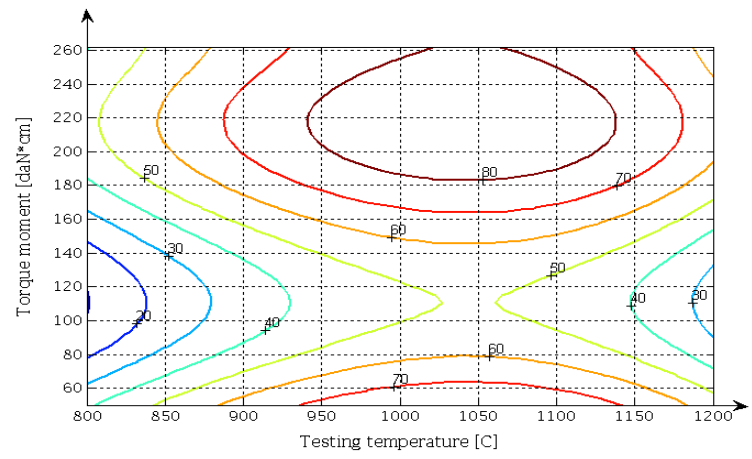


(c)

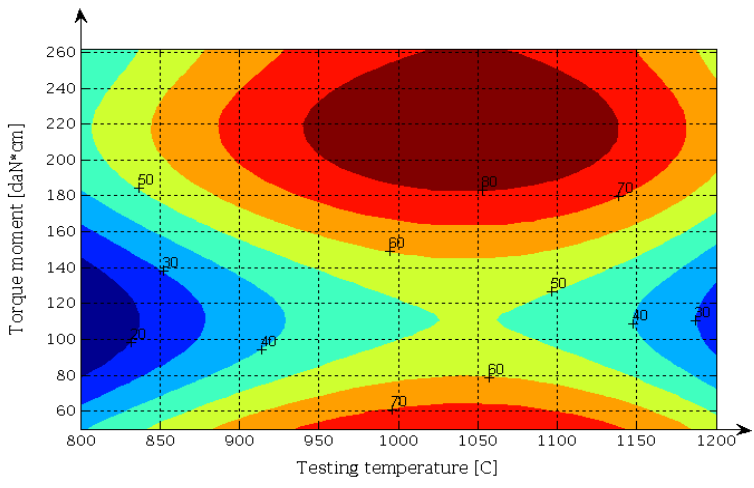

Figure 13

Diagrams for the steel specimens (lot 3), at the testing temperature (heating between $800-1200^{\circ} \mathrm{C}$ )

(a) the variation of deformation resistance, described by the torque moment value [equation type: $\mathrm{z}=\mathrm{a}_{1}$ $+\mathrm{a}_{2} \mathrm{x}+\mathrm{a}_{3} \mathrm{x}^{2}+\mathrm{a}_{4} \mathrm{x}^{3}+\mathrm{a}_{5} \mathrm{y}+\mathrm{a}_{6} \mathrm{y}^{2}+\mathrm{a}_{7} \mathrm{y}^{3}+\mathrm{a}_{8} \mathrm{y}^{4}+\mathrm{a}_{9} \mathrm{y}^{5}$, standard deviation: $\mathrm{r}^{2}=0.8287, \mathrm{a}_{1}=0.0002 ; \mathrm{a}_{2}=-$ $\left.0.7729 ; \mathrm{a}_{3}=0.0016 ; \mathrm{a}_{4}=-7.9719 ; \mathrm{a}_{5}=2.8268 ; \mathrm{a}_{6}=-0.0707 ; \mathrm{a}_{7}=0.0006 ; \mathrm{a}_{8}=-2.2391 ; \mathrm{a}_{9}=2.8577\right]$; (b), (c) the technological domains area of deformation resistance, described by the experimental torque moment value

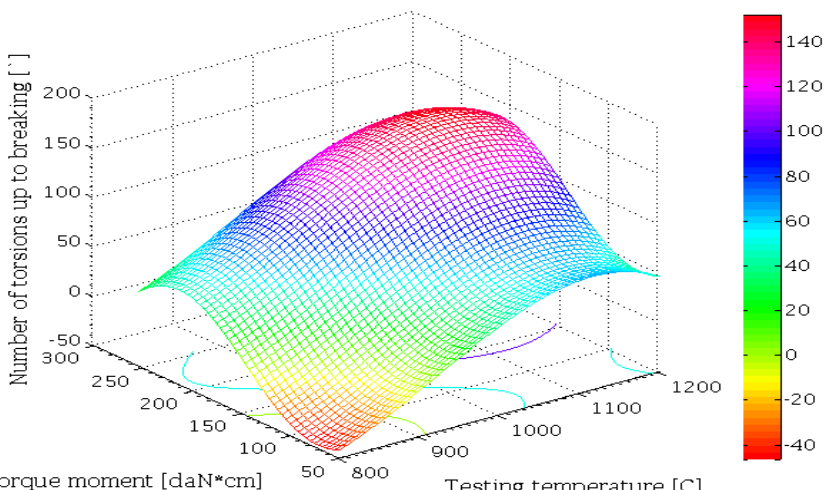

(a)

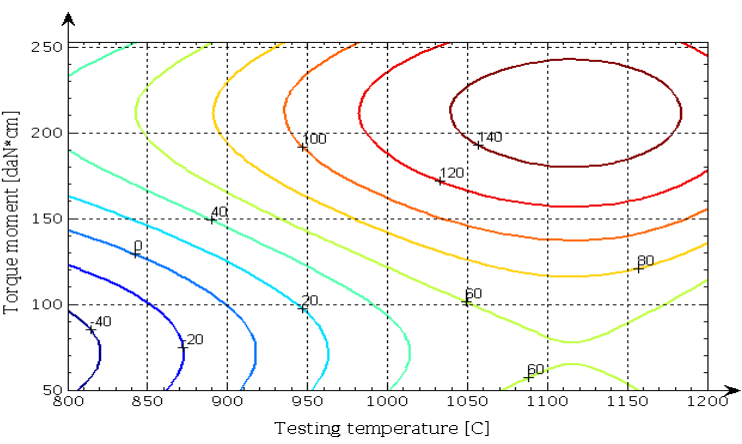

(b) 


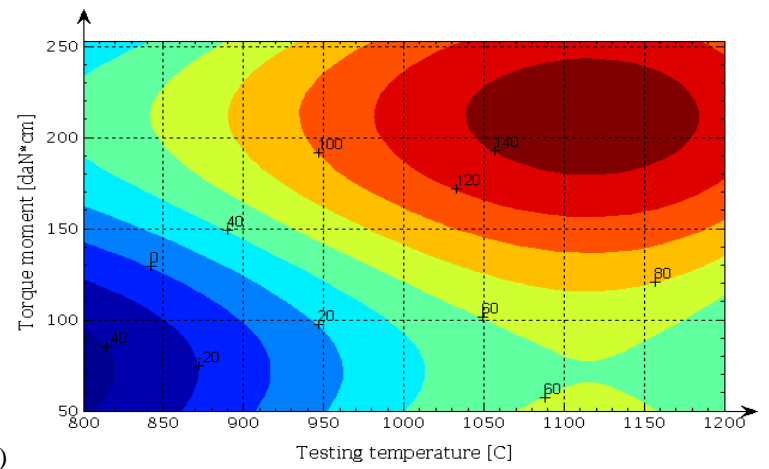

Figure 14

Diagrams for the steel specimens (lot 4), at the testing temperature (heating between $800-1200^{\circ} \mathrm{C}$ )

(a) the variation of deformation resistance, described by the torque moment value [equation type: $\mathrm{z}=\mathrm{a}$ $+\mathrm{a}_{2} \mathrm{x}+\mathrm{a}_{3} \mathrm{x}^{2}+\mathrm{a}_{4} \mathrm{x}^{3}+\mathrm{a}_{5} \mathrm{y}+\mathrm{a}_{6} \mathrm{y}^{2}+\mathrm{a}_{7} \mathrm{y}^{3}+\mathrm{a}_{8} \mathrm{y}^{4}+\mathrm{a}_{9} \mathrm{y}^{5}$, standard deviation: $\mathrm{r}^{2}=0.8511, \mathrm{a}_{1}=2645.1552 ; \mathrm{a}_{2}$ $=-9.4335 ; \mathrm{a}_{3}=0.0107 ; \mathrm{a}_{4}=-3.8952 ; \mathrm{a}_{5}=-0.0935 ; \mathrm{a}_{6}=-0.0194 ; \mathrm{a}_{7}=0.0003 ; \mathrm{a}_{8}=-1.2939 ; \mathrm{a}_{9}=$ 1.7628];

(b), (c) the technological domains area of deformation resistance, described by the experimental torque moment value

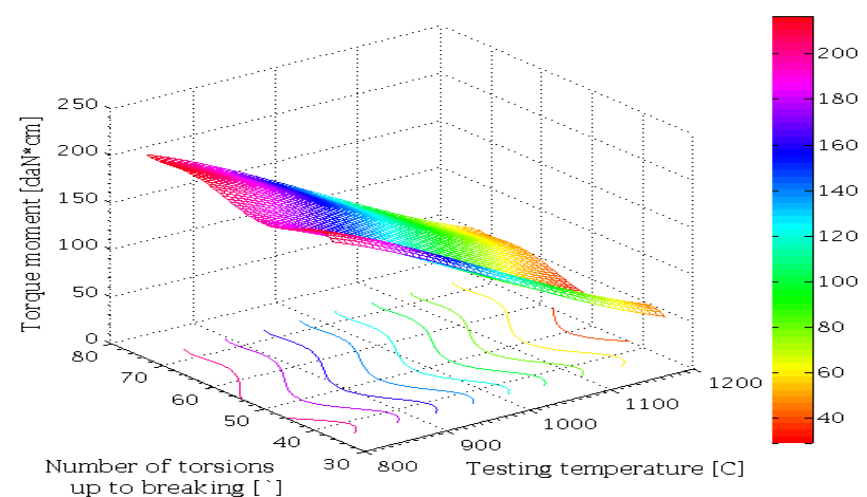

(a) up to breaking [

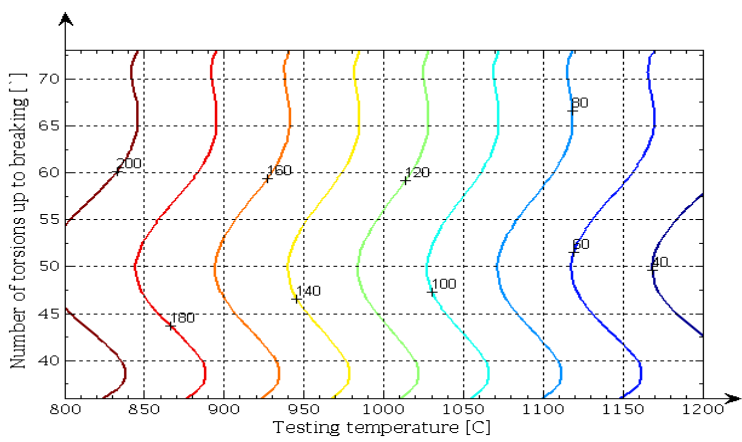

(b) 
(c)

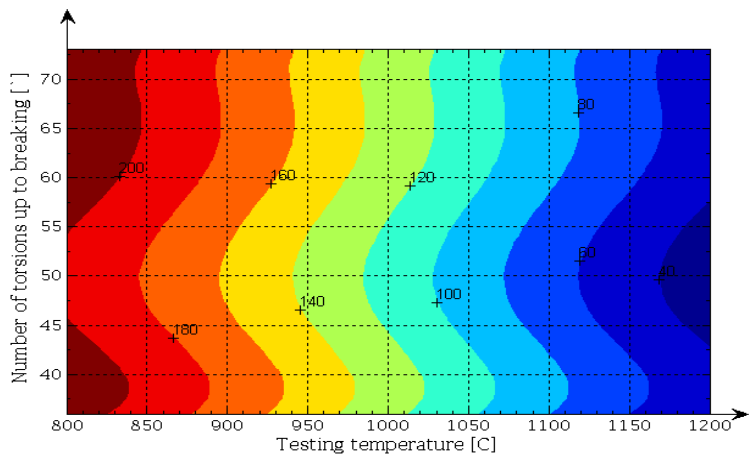

Figure 15

Diagrams for the steel specimens (lot 1), at the testing temperature (heating between $800-1200^{\circ} \mathrm{C}$ )

(a) the variations of plasticity, described by the number of torsions up to breaking [equation type: $\mathrm{z}=$ $a_{1}+a_{2} x+a_{3} x^{2}+a_{4} x^{3}+a_{5} y+a_{6} y^{2}+a_{7} y^{3}+a_{8} y^{4}+a_{9} y^{5}$, standard deviation: $r^{2}=0.9886, a_{1}=-$

$12388.9786 ; \mathrm{a}_{2}=2.9529 ; \mathrm{a}_{3}=-0.0034 ; \mathrm{a}_{4}=1.1348 ; \mathrm{a}_{5}=1138.5959 ; \mathrm{a}_{6}=-42.8862 ; \mathrm{a}_{7}=0.7911 ; \mathrm{a}_{8}=$ $\left.-0.0072 ; a_{9}=2.5444\right]$;

(b), (c) the technological domains area of plasticity, described by the experimental number of torsions up to breaking

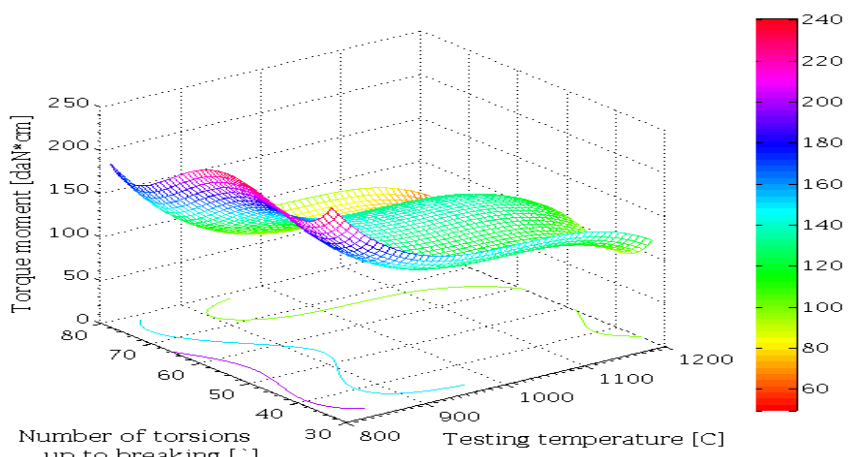

(a) up to breaking ["]

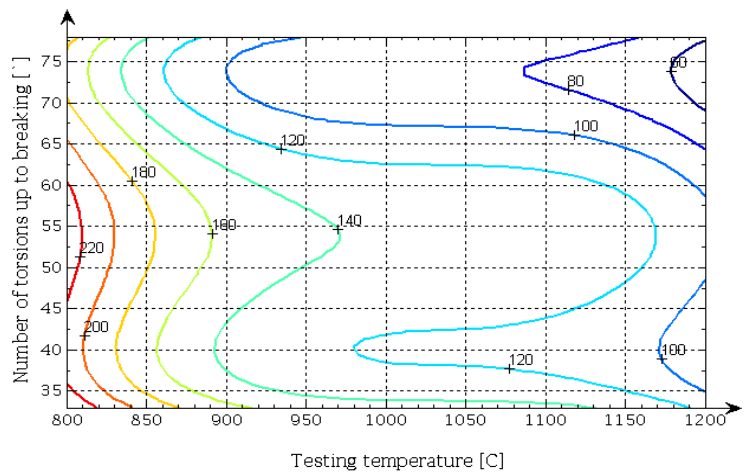

(b) 


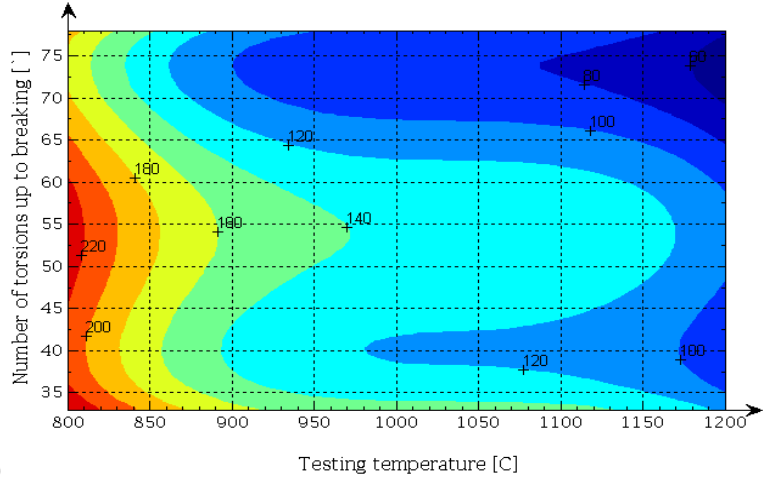

Figure 16

Diagrams for the steel specimens (lot 2), at the testing temperature (heating between $800-1200^{\circ} \mathrm{C}$ )

(a) the variations of plasticity, described by the number of torsions up to breaking [equation type: $\mathrm{z}=$ $\mathrm{a}_{1}+\mathrm{a}_{2} \mathrm{x}+\mathrm{a}_{3} \mathrm{x}^{2}+\mathrm{a}_{4} \mathrm{x}^{3}+\mathrm{a}_{5} \mathrm{y}+\mathrm{a}_{6} \mathrm{y}^{2}+\mathrm{a}_{7} \mathrm{y}^{3}+\mathrm{a}_{8} \mathrm{y}^{4}+\mathrm{a}_{9} \mathrm{y}^{5}$, standard deviation: $\mathrm{r}^{2}=0.9923, \mathrm{a}_{1}=10758.4402 ;$ $\mathrm{a}_{2}=-22.3451 ; \mathrm{a}_{3}=0.0215 ; \mathrm{a}_{4}=-6.9343 ; \mathrm{a}_{5}=-217.5808 ; \mathrm{a}_{6}=5.6907 ; \mathrm{a}_{7}=-0.0567 ; \mathrm{a}_{8}=7.0028 ; \mathrm{a}_{9}=$ $1.2923]$

(b), (c) the technological domains area of plasticity, described by the experimental number of torsions up to breaking

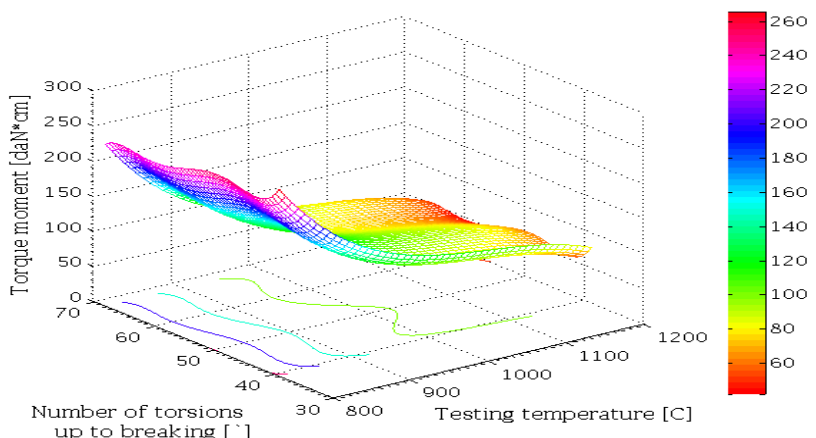

(a) up to breaking ["]

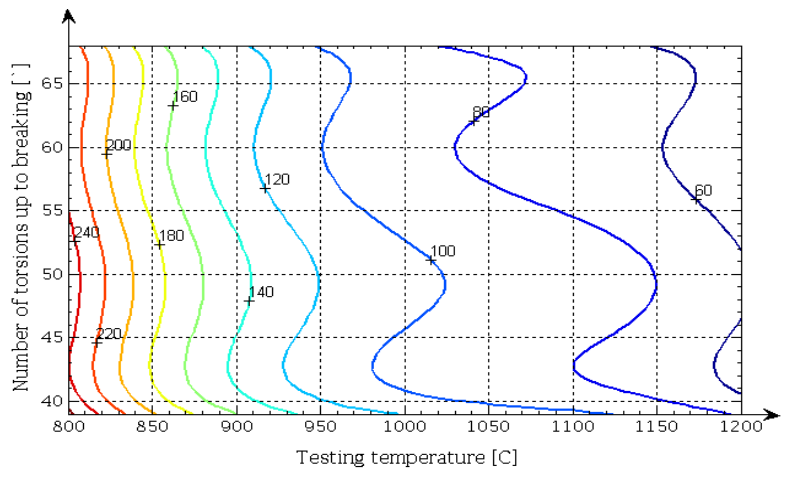

(b) 


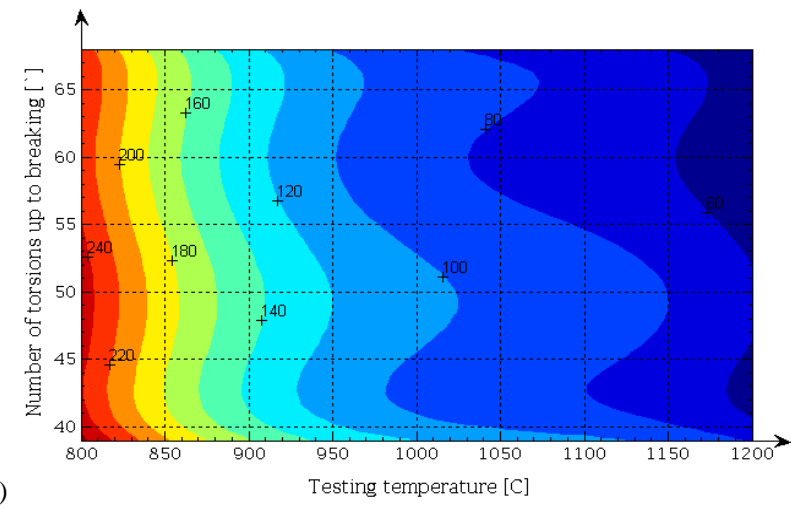

Figure 17

Diagrams for the steel specimens (lot 3), at the testing temperature (heating between $800-1200^{\circ} \mathrm{C}$ )

(a) the variations of plasticity, described by the number of torsions up to breaking [equation type: $\mathrm{z}=$ $a_{1}+a_{2} x+a_{3} x^{2}+a_{4} x^{3}+a_{5} y+a_{6} y^{2}+a_{7} y^{3}+a_{8} y^{4}+a_{9} y^{5}$, standard deviation: $r^{2}=0.9922, a_{1}=73428.8648 ;$ $\mathrm{a}_{2}=-21.6111 ; \mathrm{a}_{3}=0.0201 ; \mathrm{a}_{4}=-6.2614 ; \mathrm{a}_{5}=-6257.4592 ; \mathrm{a}_{6}=236.7125 ; \mathrm{a}_{7}=-4.4353 ; \mathrm{a}_{8}=0.0412 ;$

$$
\left.\mathrm{a}_{9}=-0.0002\right] \text {; }
$$

(b), (c) the technological domains area of plasticity, described by the experimental number of torsions up to breaking

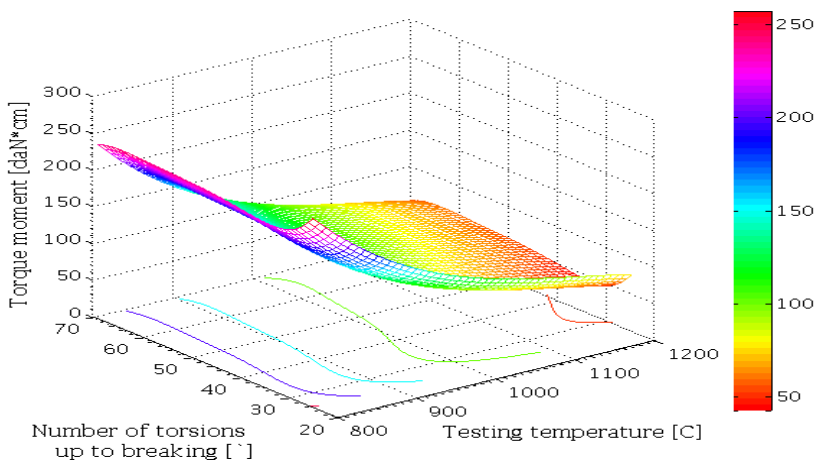

(a) up to breaking [']

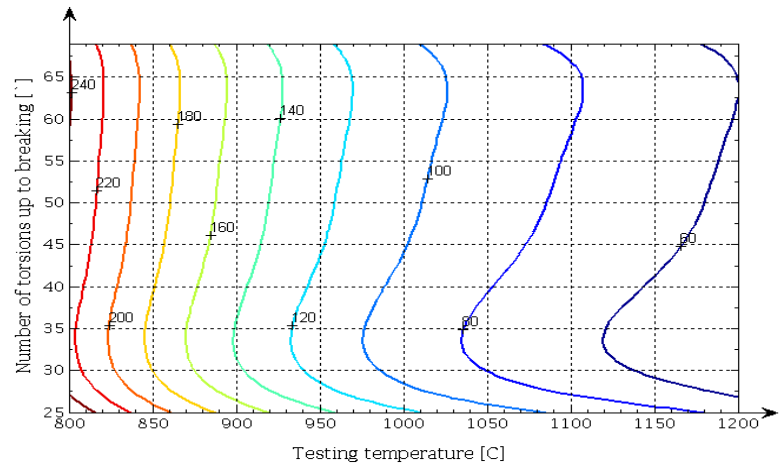

(b) 


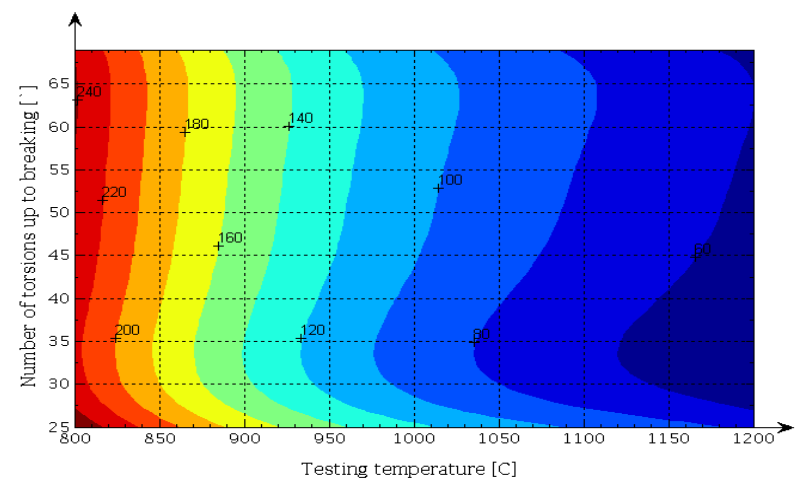

(c)

Figure 18

Diagrams for the steel specimens (lot 4), at the testing temperature (heating between $800-1200^{\circ} \mathrm{C}$ )

(a) the variations of plasticity, described by the number of torsions up to breaking [equation type: $\mathrm{z}=$ $\mathrm{a}_{1}+\mathrm{a}_{2} \mathrm{x}+\mathrm{a}_{3} \mathrm{x}^{2}+\mathrm{a}_{4} \mathrm{x}^{3}+\mathrm{a}_{5} \mathrm{y}+\mathrm{a}_{6} \mathrm{y}^{2}+\mathrm{a}_{7} \mathrm{y}^{3}+\mathrm{a}_{8} \mathrm{y}^{4}+\mathrm{a}_{9} \mathrm{y}^{5}$, standard deviation: $\mathrm{r}^{2}=0.9879, \mathrm{a}_{1}=6295.2783$; $\mathrm{a}_{2}=-10.4354 ; \mathrm{a}_{3}=0.0090 ; \mathrm{a}_{4}=-2.6546 ; \mathrm{a}_{5}=-227.7726 ; \mathrm{a}_{6}=9.4815 ; \mathrm{a}_{7}=-0.1935 ; \mathrm{a}_{8}=0.0019 ; \mathrm{a}_{9}=$ $-7.7299]$

(b), (c) the technological domains area of plasticity, described by the experimental number of torsions up to breaking

\section{Discussions}

Regarding the hot torsion testing, we have the following remarks:

- As indicated in the research line, 4 lots of low alloyed medium carbon steel grades (grade 43MoMn16 and grade 33MoCr11) were developed, as shown in Table 2. From these steels the test samples were made, in order to determine the deformability characteristics (plasticity and deformation resistance);

- Thus, 108 specimen-samples belonging to the 4 lots were made and subjected to the hot torsion tests, in an own facility equipped with a tubular oven, at the testing temperature (heating being between $800-1200^{\circ} \mathrm{C}$ ). Each determination has been repeated three times, and on the same number of test samples. The results are presented in Tables 3-6.

In the graphical representation of the hot torsion tests results, presented in Figures 9-10, we have the following comments and remarks:

- The cumulative variations presented in Figures 9-10 are shown as quantitative dependencies, based on the results shown in Tables 3-6; 
- The variation of torque moment (variation of deformation resistance) in the case of the 4 experimental lots of steels, at the experimental testing temperature (heating between $800-1200^{\circ} \mathrm{C}$ ) are presented cumulative in the Figure 9. The variations, as shown in the above mentioned figures, indicate that the deformation resistance decreases with increasing the heating temperature;

- The number of torsions up to breaking (variations of plasticity) in the case of the 4 experimental lot of steels, at the experimental testing temperature (heating between $800-1200^{\circ} \mathrm{C}$ ) are presented cumulative in Figure 10.

- It is observed a clear similarity between the characteristics of the analyzed steels, being higher in cases of lots 3 and 4 . This explanation consists in the fact that, by increasing the alloying degree, the crystallization speed decreases, which means that loss of plasticity, especially close to the $900^{\circ} \mathrm{C}$. Although the steel in the lot 3 is allied with Chrome and Molybden, the level of the characteristics of plasticity is lower but constant, with a slight increase at temperature, in the range of $1100-1200^{\circ} \mathrm{C}$ compared to steels in lots 1 and 2 .

- We note that the analyzed steel grades show lower plasticity under $900^{\circ} \mathrm{C}$, when the deformation resistance increases considerably, which is more evident in cases of lots 3 and 4 . Between $900-1200^{\circ} \mathrm{C}$, the plasticity is good and it increases with the temperature increases. The resistance at the deformation is not too high.

- A higher amount of Sulphur is available for steel in lot 3, which deserves a specific analysis. As this quantity is much greater than lots 1 and 2 or even the lot 4 , a comparative analysis of these should be performed, indicating the effect on the deformation of the Sulphur content which forms more or less complex inclusion. These results relate quite well to current trends in the steels destined to the seamless pipe manufacturing, and impose steels for these purposes a certain Sulphur content, in view to increasing the plasticity.

Regarding the regression analysis used for observation of the deformation resistance of the 4 lots of low alloyed medium carbon steel grades, we have the following comments:

- The variations of deformation resistance, described by the torque moment value, are presented in Figure 11-14 (a), determined by Matlab, using the regression analysis [equation type: $z=a_{1}+a_{2} x+a_{3} x^{2}+a_{4} x^{3}+a_{5} y+a_{6} y^{2}+a_{7} y^{3}$ $+\mathrm{a}_{8} \mathrm{y}^{4}+\mathrm{a}_{9} \mathrm{y}^{5}$, with standard deviation $\mathrm{r}^{2}$ between $0.8287-0.9764$;

- The technological domains area of deformation resistance, described by the torque moment value represents the deformability diagrams for the steel specimens (lots 1-4), at the experimental testing temperature (heating between $800-1200^{\circ} \mathrm{C}$ ). These diagrams are presented in Figure 11-14 (b)-(c). 
- The relationships that determine the technological areas are useful because they can indicate a predictive relationship that can be exploited in practice.

Regarding the regression analysis used for observation of the plasticity of the 4 lots of low alloyed medium carbon steel grades, we have the following comments:

- The variations of plasticity, described by the number of torsions up to breaking, are presented in Figure 15-18 (a), determined by Matlab, using the regression analysis [equation type: $\mathrm{z}=\mathrm{a}_{1}+\mathrm{a}_{2} \mathrm{x}+\mathrm{a}_{3} \mathrm{x}^{2}+\mathrm{a}_{4} \mathrm{x}^{3}+\mathrm{a}_{5} \mathrm{y}+\mathrm{a}_{6} \mathrm{y}^{2}+\mathrm{a}_{7} \mathrm{y}^{3}$ $+\mathrm{a}_{8} \mathrm{y}^{4}+\mathrm{a}_{9} \mathrm{y}^{5}$, with standard deviation $\mathrm{r}^{2}$ between $\left.0.9879-0.9986\right]$;

- The technological domains area of plasticity, described by the number of torsions up to breaking represents the deformability diagrams for the steel specimens (lots 1-4), at the experimental testing temperature (heating between $\left.800-1200^{\circ} \mathrm{C}\right)$. These diagrams are presented in Figure 15-18 (b)-(c).

Having in view the experimental results described above, we can conclude the followings:

- The temperature range at which deformation is to be made must be determined first if an optimal heat condition is chosen. This temperature range is specific to each material and is dependent on the size of the degree of deformation that can be made without apparition of any cracks;

- The allowed temperature range is set so that the deformation resistance is low and the high deformation is achieved. In terms of resistance to deformation, the trend would be that the upper-temperature limit is as high as possible. For the purpose of deformations this limit is fixed at that temperature at which the deformability begins to fall;

- The technological temperature range shall be the interval for plastic deformation and shall be within the permissible temperature range. This range must be determined on the basis of correlation diagrams drawn follow the technological tests which determine the deformability of steel;

- The upper limit of the optimum value of heating temperatures applied for deforming both low alloyed medium carbon steel grades, results clearly from the correlation diagrams, is $950^{\circ} \mathrm{C}$;

- Regarding the end heating temperature, for the hot deformation of the low alloyed medium carbon steel grades, we have the following experimental values (or ranges):

$\equiv 1150-1200^{\circ} \mathrm{C}$, for grade $43 \mathrm{MoMn} 16$;

$\equiv 1150^{\circ} \mathrm{C}$, for grade $33 \mathrm{MoCr} 11$;

- Thus, from the hot torsion tests carried out to determine the hot deformability, it results that the optimal plasticity of the analyzed steels is found within the temperature range $950-1200^{\circ} \mathrm{C}$. The interpretation of the deformation diagrams 
leads to the conclusion that the optimum plasticity for plastic deformation by hot rolling of the analyzed 4 lots is recommended in a range of $950-1200^{\circ} \mathrm{C}$;

- Starting from the temperature of $900^{\circ} \mathrm{C}$, both steel grades from the four lots have sufficient plasticity, but the value of the deformation resistance is still high up to the temperature of $950^{\circ} \mathrm{C}$. The growth dynamic of the plasticity characteristics is continuous, reaching the maximum value at the temperature of $1200^{\circ} \mathrm{C}$, while the resistance to deformation is reduced.

\section{Conclusions}

Hot-rolled seamless steel pipe is defined as a wrought steel tubular product made without a welded seam, manufactured by hot-rolling. It is used for conveying gas, water, and petroleum of both oil and natural gas industries. This study includes the results of the experimental tests conducted to find the plasticity and deformability characteristics of two low alloyed medium carbon steel grades (EA < 2.5\%) destined for seamless tubes and pipes manufacturing and used in the petroleum industry: grade 43MoMn16 and grade 33MoCr11.

Existing standards in the oil and gas industry regulate the physico-mechanical characteristics and test patterns of steels from which the hot rolled seamless steel pipes and tubes are used, framing them in some classes of resistance. The composition of steels, their method of elaboration, and the thermal treatments corresponding to these classes are left at the discretion of the manufacturers. Therefore, the knowledge about the characteristics of deformability has for the technologist, as well as for the designer and researcher, a great practical significance, because they are important elements in establishing a correct technological process. The indications regarding the variation of plasticity with the temperature, using the hot torsion method, allowed for establishing the temperature range within which the steel plasticity is optimal and in which, in general, it is recommended to perform the entire hot plastic deformation.

The study of deformations according to the temperature (and the chemical composition too), on test lots, has established useful conclusions for the practice of rolling of these grades (43MoMn16 and 33MoCr11). The determination of hot plastic deformations values, correlated with the results of several structural research, may constitute the basic parameters for the rational development of the rolling processes.

\section{Acknowledgement}

The equipment destined to study the steels deformability by hot torsion (property of the Faculty of Engineering Hunedoara), is subject to a Romanian patent, entitled "Facility adapted for experimental determination of the resistance to thermal fatigue of samples placed tangentially on the generator of support discs," registered in the Romanian State Office for Inventions and Trademarks (OSIM). 


\section{References}

[1] Reports and Data - Market News, 2019, https://www.reportsanddata.com

[2] A. Yamane, H. Shitamoto, K. Yamane, Development of numerical analysis on seamless tube and pipe process, Nippon Steel \& Sumitomo Metal Technical Report, 107 (2015), pp. 108-113

[3] K. Masamura, Y. Nagahama, Manufacturing processes and products of steel pipes and tubes in JFE steel, JFE Technical Report, 7 (2006), pp. 1-6

[4] D. Kuc, G. Niewielski, Technological plasticity and structure in stainless steels during hot-working, Journal of Achievements in Materials and Manufacturing Engineering, 32, 2 (2009), pp. 154-161

[5] Z. Pater, J. Kazanecki, Complex numerical analysis of the tube forming process using diescher mill, Archives of Metallurgy and Materials, 58/3 (2013), pp. 717-724

[6] J. Magaone, I. Ilca, Influence factors analysis and steels behavior within the high temperature operating, Ştiinţa şi Inginerie, 16 (2009), pp. 629-636

[7] V. Alexa, I. Kiss, Experimental tests on the plasticity and deformability characteristics of several stainless steel grades used for hydro-pneumatic equipment's manufacturing, Acta Polytechnica Hungarica, 15, 8 (2018), pp $121-139$

[8] V. Alexa, I. Kiss, Deformability of martensitic stainless steel (grade X46Cr13) by hot torsion, Annals of Faculty Engineering Hunedoara International Journal of Engineering, XVII, 2 (2019), pp. 209-214

[9] I. Ilca, I. Kiss, V. Alexa, S. Rațiu, Analysis of some steel behaviour at high operating temperatures, Annals of Faculty of Engineering HunedoaraInternational Journal of Engineering, 14, 2 (2016), pp. 183-189

[10] STAS 8185-88: Steels for seamless tubes for petroleum industry. Grades and technical requirements for quality

[11] DIN EN 10297-1: Seamless circular steel tubes for mechanical and general engineering purposes; Non-alloy and alloy steel tubes 